\title{
Development of UV-Reactive Electrospinning Method Based on Poly(ethylene glycol) diacrylate Crosslinking
}

\begin{abstract}
Electrospinning is a popular method for creating nonwoven fiber materials for a wide variety of applications. In the field of biomaterials, electrospun materials are favoured because of a high surface-to-volume ratio which can be useful for drug loading and release, and because nanoscale fibers mimic native tissue structures, improving cell interactions. However limitations exist with regards to traditional solvent evaporation-based electrospinning techniques.
\end{abstract}

A new area of research into reactive electrospinning is investigating methods of electrospinning that rely on in situ crosslinking rather than solvent evaporation to stabilize fibers. These techniques can potentially reduce the waste of excess solvents and make it easier to electrospin water soluble polymers.

In this work, UV photocrosslinked PEGDA is evaluated as a material for reactive electrospinning. To facilitate the electrospinning process poly(ethylene glycol) diacrylate (PEGDA) is combined with polyvinyl alcohol (PVA). PEGDA/PVA solutions can be successfully electrospun under constant UV light exposure to initiate the crosslinking of the PEGDA. Reactive electrospun fibers appear more stable immediately after spinning and after washing with water, indicating successful photo crosslinking.

Keywords: reactive electrospinning, PEGDA, UV crosslinking

https://doi.org/10.1515/cdbme-2020-3048

\footnotetext{
${ }^{*}$ Corresponding author: Jennifer Huling: Institute for Biomedical Engineering, Rostock University Medical Center, Friedrich-Barnewitz-Str. 4, 18119 Rostock, Germany, e-mail: Jennifer.huling@uni-rostock.de Beate Lyko, Sabine IIIner, Nicklas Fiedler, Niels Grabow and Michael Teske: Institute for Biomedical Engineering, Rostock University Medical Center, Rostock, Germany
}

\section{Introduction}

Electrospinning as a method of reproducibly creating nonwoven fiber mats of micro- to nano-sized fibers for application in textile, industrial and medical applications has been well-studied [1]. Traditionally, polymers are dissolved in highly volatile solvents that evaporate during the fiber time-of-flight to create stable polymer fibers, but this method does have some limitations. Usually electrospun polymer solutions are mostly composed of the solvent, which is ultimately lost via evaporation during spinning. Additionally, these solvents are often not environmentally friendly and can limit biocompatibility if any toxic residuals remain after processing [1].

As a result, there has been a shift in research to examine alternative ways to stabilize fibers that are not dependent on solvent evaporation. One broad category of these methods is known as reactive electrospinning or in situ crosslinking. The main idea behind reactive electrospinning is to crosslink during the spinning process. The crosslinking most often occurs during the flight of the polymer between the needle and the collector. Methods for achieving this include thermal, chemical and photo crosslinking during the electrospinning process [1]. One strategy, ultra violet (UV) photo-curing reactive electrospinning, is popular because it is compatible with a wide range of polymers and UV light is relatively easy to add to existing electrospinning setups. UV photo-curing reactive electrospinning methods have been developed for a variety of polymer solutions [2,3]. Typically, polymer solutions consist of a photo crosslinkable monomer, a photoinitiator and an additional copolymer or carrier polymer. Here we investigate the development of a UVreactive electrospinning method based on the in situ crosslinking of poly(ethylene glycol) diacrylate (PEGDA). Polyvinyl alcohol (PVA) was used as a carrier molecule to facilitate electrospinning. The PEGDA/PVA nonwoven fibers made under reactive electrospinning conditions had a 
normal morphology and were more stable in water than those made without UV exposure.

\section{Materials and Methods}

\subsection{General Information}

PEGDA with molecular weight 250 and $700 \mathrm{~g} / \mathrm{mol}$ (PEGDA $_{250}$ and PEGDA $_{700}$, respectively), PVA $(31,000-$ $50,000 \mathrm{~g} / \mathrm{mol})$ and the photoinitiator (PI) 2-hydroxy-4'- (2hydroxyethoxy) -2-methyl propiophenone (Irgacure 2959) were purchased from Sigma-Aldrich (Munich, Germany). Dimethyl sulfoxide (DMSO) was purchased from Carl Roth (Karlsruhe, Germany). A $50 \% \mathrm{w} / \mathrm{v}$ stock solution of PI in DMSO was prepared and used in all experiments. PI stock solution was mixed directly with PEGDA solutions immediately before use to generate solutions with final PI concentrations of 5, 10 and $20 \% \mathrm{w} / \mathrm{w}$ (relative to the mass of PEGDA).

\subsection{FTIR measurements}

FTIR-ATR-measurements were performed using a Vertex 70 IR-Spectrometer (Bruker Corporation) with a Golden Gate ${ }^{\mathrm{TM}}$ (Specac Limited) ATR diamond insert. A spectrum was recorded at a rate of 1 measurement/second using OPUS spectroscopy software (Bruker Corporation). For each sample, $5 \mu \mathrm{L}$ of solution was pipetted onto the measurement surface. The $10 \mathrm{~mW} 365 \mathrm{~nm}$ LED UV light source (Ocean Insight, Ostfildern, Germany) was suspended approximate $4.5 \mathrm{~cm}$ away from the sample using a custom made cover. The FTIR measurements and UV light exposure at 100\% power were started simultaneously. Peak areas between 1605 and $1651 \mathrm{~cm}^{-1}$ were used to estimate the number of double bonds in the sample. The reaction was considered complete when the change in peak area plateaued. PEGDA 250 and PEGDA $_{700}$ with $5 \%$ and $10 \%(\mathrm{w} / \mathrm{w})$ PI were analysed (each group had $n=3$ samples). Means were compared with ANOVA and Bonferroni post-test. $\mathrm{p}<0.05$ was considered significant (GraphPad Prism).

\subsection{Reactive electrospinning}

Solutions for electrospinning were made by dissolving $15 \%$ w/w PVA and various amounts of PEGDA 700 in water. Solutions were mixed at $37^{\circ} \mathrm{C}$ for at least 12 hours. PEGDA solutions of $2.5,5,7.5$ and $10 \% \mathrm{w} / \mathrm{w}$ polymer were tested.
Immediately before electrospinning, PI was added to reach a final concentration of $20 \% \mathrm{w} / \mathrm{w}$.

Electrospinning was performed with a Contipro (Dolní Dobrouč, Czech Republic) 4SpinC4S LAB2 device. A solution flow rate of $16 \mu \mathrm{L} / \mathrm{min}$, collector distance of $25 \mathrm{~cm}$ and applied voltage of $35 \mathrm{kV}$ was used in a $22^{\circ} \mathrm{C}$ and $24 \%$ humidity environment. UV crosslinking was initiated during the electrospinning process by exposing the area near the needle to $365 \mathrm{~nm}$ UV light (Fig 1A). A custom-made device held the light source and allowed for distance and angle adjustments (Fig 1B). The UV light source was used at $100 \%$ power and a distance of $18.5 \mathrm{~cm}$ and angle of $65^{\circ}$ from the tip of the needle. After $30 \mathrm{~min}$ of spinning, nonwoven fiber mats were placed in a UV chamber (UV-Ofen CL-1000 Ultraviolet Crosslinker, Ultra-Violet Products Ltd., Cambridge, UK) for $10 \mathrm{~min}$ under $365 \mathrm{~nm}$ light.
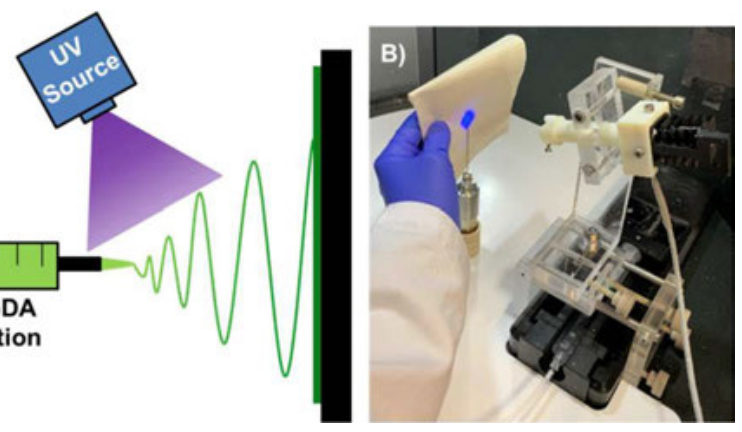

Figure 1: (A) The schematic diagram shows the general UVreactive electrospinning setup. During the electrospinning process, UV light is used to initiate crosslinking of PEGDA. (B) Pictured is the set-up used to complete this work. A custom-designed holder was utilized to control of the distance and angle of the UV light.

\subsection{SEM imaging}

Scanning electron microscopy (SEM) was performed on a Quanta FEG 250 (FEICompany, Germany). Nonwoven fiber samples were cut and mounted on aluminium carriers using graphite tape before being imaged. Samples were imaged once directly after spinning, then washed $3 \mathrm{x}$ in deionized water with a pipette, soaked for 5 additional minutes, rinse $3 x$ again, dried in vacuum and imaged again. 


\section{Results and Discussion}

\subsection{Relative Crosslinking Speed}

FTIR measurements were used to monitor the loss of double bonds in PEGDA solutions while undergoing UV crosslinking. The crosslinking reaction was considered to be complete when the conversion of the double bonds stopped according to section 2.2. By monitoring the amount of time until the completion of the crosslinking reaction, the relative speed of the crosslinking reaction was compared between: 250 and $700 \mathrm{~g} / \mathrm{mol}$ PEGDA solutions with 5 or $10 \% \mathrm{w} / \mathrm{w}$ photoinitiator. ANOVA analysis showed no significant affect from photoinitiator concentration, but the molecular weight of PEGDA did have a significant effect on the speed of the crosslinking reaction (Fig. 2), revealing that PEGDA $_{700}$ finished crosslinking significantly faster than $\mathrm{PEGDA}_{250}$. In situ crosslinking during electrospinning would ideally result in complete or nearly complete crosslinking before fibers reach the collector to ensure stable fibers and minimal fiber fusion. Time-of-flight for fibers is estimated to be less than 0.5 seconds, indicating the crosslinking speed is a critical factor and that polymer solutions should be optimized to have the fastest crosslinking speed possible [4]. For this reason, all further experiments were carried out using PEGDA $_{700}$. Following the demonstrated pattern, higher molecular weight PEGDA solutions might have even faster crosslinking times, but we were unable to test them due to low solubility in our testing conditions.

\subsection{UV reactive electrospinning}

While the faster of the PEGDA polymers tested was higher in molecular weight, it was still too low for ideal electrospinning. PEGDA 700 only solutions were unable to be electrospun (data not shown). To compensate for the relatively low molecular weight of the PEGDA, a carrier polymer was included for the purpose of electrospinning. The formation of fibers during the electrospinning process has been linked to a critical threshold of viscosity related to molecular entanglement [5]. More molecular entanglement leads to better formation of fibers rather than droplets during spinning. Therefore, larger carrier molecules can be added to low molecular weight polymers to facilitate electrospinning. PVA was chosen as a carrier polymer because it has been used as a carrier with other small polymers, is biocompatible and is soluble in water [6,7].
Nonwoven fiber mats were electrospun with increasing amounts of PEGDA 700 relative to a constant amount of PVA. Representative SEM images from the highest and lowest tested PEGDA concentrations, with and without UV exposure are shown in Figure 3. All samples exhibit fiber formation. In $10 \%$ PEGDA samples there is a noticeable difference between samples with and without UV exposure. Without UV exposure, the fibers appear fused together, while the samples created with UV exposure has well formed, distinct fibers. This observation could be a sign of successful in situ crosslinking during time-of-flight. Crosslinking during

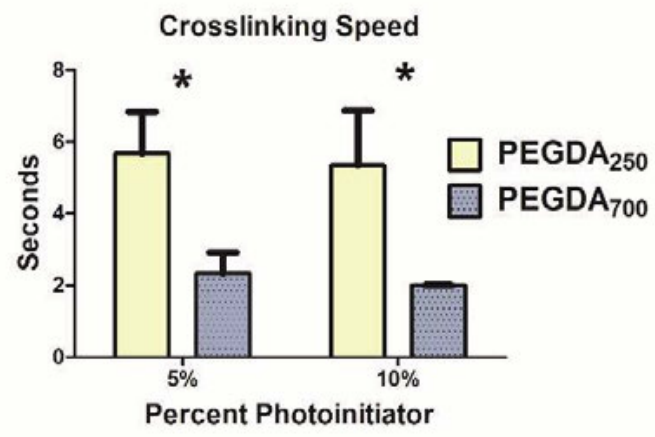

Figure 2: FTIR was used to measure the conversion of double bonds in PEGDA solutions. The graph displays mean time to completion of the crosslinking reaction and standard deviations. $\left({ }^{*}\right)$ denotes significant differences between $\mathrm{PEGDA}_{250}$ and PEGDA 700 samples at the same photoinitiator concentration $(p<0.05)$.

electrospinning may be stabilizing the fibers before collection, preventing the fibers from fusing after deposition.

Prepared nonwoven mats were washed with deionized water briefly to further assess the materials stability. SEM images of representative nonwovens after washing are shown in Figure 4. At both PEGDA concentrations, UV exposure had a significant effect on the stability of the material in water. The UV-exposed samples have retained a generally fibrous structure while the samples that were not exposed to UV light were either completely or mostly dissolved, leaving little visible material behind. Because both PVA and PEGDA are water soluble, this experiment demonstrates that crosslinking did occur with UV exposure. PVA does not directly crosslink with the PEGDA, but rather should form an interpenetrating network with the crosslinked PEGDA [6]. Further work will need to be done to assess whether PVA is still present after washing or if it is largely removed, leaving PEGDA-only fibers.

The proof-of-concept work done in this paper demonstrated the feasibility of using reactive electrospinning to create in situ crosslinked PEGDA/ PVA nonwoven fiber materials. Future work will focus on optimizing polymer 
solutions and UV exposure parameters to maximize crosslinking during time-of-flight of the electrospinning process.

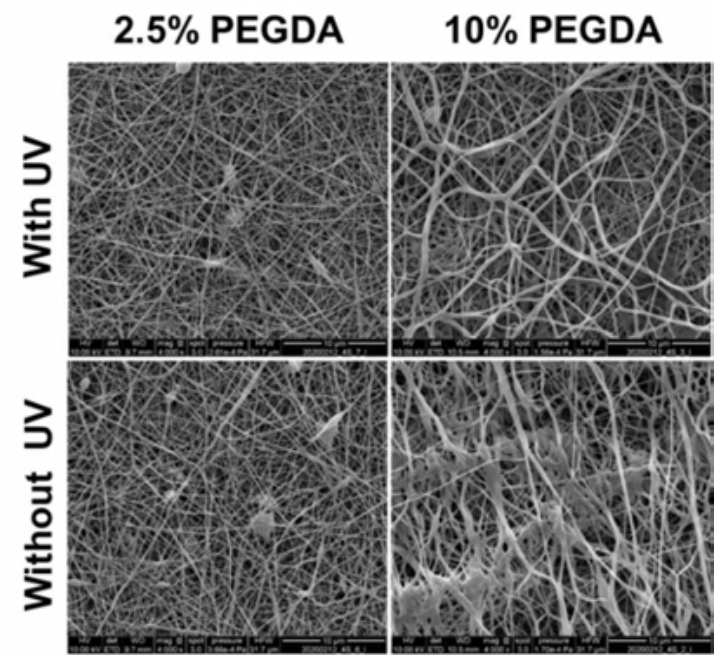

Figure 3: Representative SEM images of electrospun PVA/ PEGDA solutions with or without UV exposure. Fiber fusion can be seen in the 10\% PEGDA samples without UV exposure, but with UV the fibers are well defined. All images are at $4000 x$ magnification. Scale bars indicate $10 \mu \mathrm{m}$.

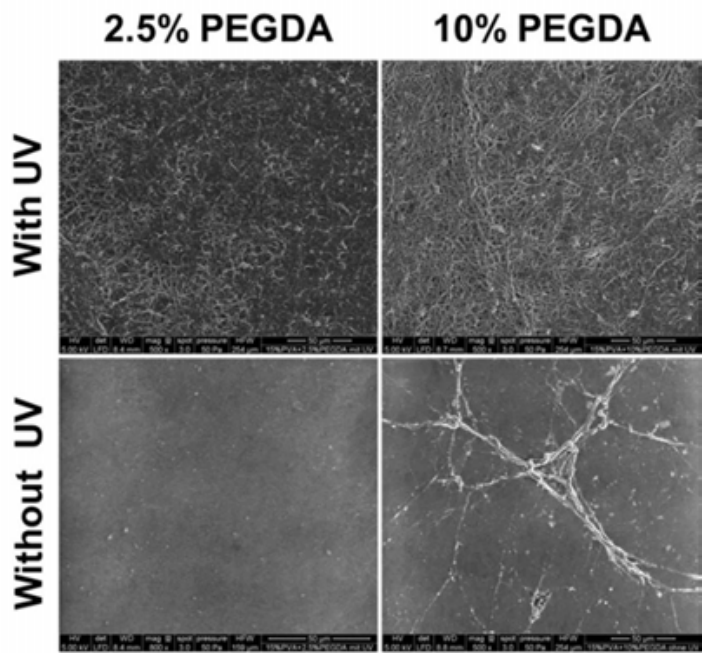

Figure 4: Representative SEM images of PVA/ PEGDA nonwovens with or without UV exposure after washing in water. Without UV, the fibers almost completely dissolve in water, while UV-exposed fibers maintain their general structure. Images are at 500 or $800 x$ magnification. Scale bars indicate $50 \mu \mathrm{m}$.

\section{Conclusion}

In this study, the feasibility of reactive electrospinning of PEGDA based on UV crosslinking was examined. PVA/PEGDA polymer blends could be electrospun to reliably produce nonwoven fiber mats without the need for volatile solvents. The addition of UV exposure simultaneously with the electrospinning process did not interfere with fiber formation. The reactive electrospun PVA/ PEGDA fibers were more stable in water than those created without UV exposure, demonstrating evidence of PEGDA crosslinking. This work establishes a method that can be used for further optimization.

\section{Author Statement}

Acknowledgements: The authors would like to thank Katja Hahn, Jonathan Ortelt, Volkmar Senz and Babette Hummel for their technical assistance and contributions. Research funding: Financial support by the Federal Ministry of Education and Research (BMBF) within RESPONSE "Partnership for Innovation in Implant Technology" is gratefully acknowledged. Conflict of interest: Authors state no conflict of interest. Informed consent: not applicable. Ethical approval: not applicable.

\section{References}

[1] Zhang B, Yan X, He HW, et al. Solvent-free electrospinning: opportunities and challenges, Polym. Chem. 2017;8: 333-352.

[2] Mehmood MF, Sangermano M, Gule NP, et al. UV Curing of Electrospun Polysulfone Fibers Containing an Acrylate as Cross-Linker, MACROMOL CHEM PHYS 2017;218.

[3] Gupta P, Trenor SR, Long TE, Wilkes GL. In Situ PhotoCross-Linking of Cinnamate Functionalized Poly(methyl methacrylate- co -2-hydroxyethyl acrylate) Fibers during Electrospinning, Macromolecules 2004;37:9211-9218.

[4] Shanmuganathan K, Elliot SM, Lane AP, Ellison CJ. Highly stretchable thermoset fibers and nonwovens using thiol-ene photopolymerization, ACS Appl. Mater. Interfaces 2014;6: 14259-14265.

[5] Shenoy SL, Bates WD, Frisch HL, Wnek GE. Role of chain entanglements on fiber formation during electrospinning of polymer solutions. Polymer 2005;46: 3372-3384.

[6] Stephens-Altus JS, Sundelacrus P, Rowland ML, West JL. Development of bioactive photocrosslinkable fibrous hydrogels. J. Biomed. Mater. Res 2011;98:167-176.

[7] Deniz DY, Kahraman MV, Kuruca SE. UV-reactive electrospinning of keratin/4-vinyl benzene boronic acidhydroxyapatite/poly(vinyl alcohol) composite nanofibers, Polym. Compos. 2015;38:1371-1377. 